\title{
BMJ Open Rates of rehospitalisation in the first 2 years among preterm infants discharged from the NICU of a tertiary children hospital in Vietnam: a follow-up study
}

\author{
Chuong Huu Thieu Do (D) , ${ }^{1}$ Malene Landbo Børresen, ${ }^{2}$ Freddy Karup Pedersen, ${ }^{2}$ \\ Ronald Bertus Geskus (D) ,3,4 Alexandra Yasmin Kruse ${ }^{2}$
}

To cite: Do CHT, Børresen ML, Pedersen FK, et al. Rates of rehospitalisation in the first 2 years among preterm infants discharged from the NICU of a tertiary children hospital in Vietnam: a follow-up study. BMJ Open 2020;10:e036484. doi:10.1136/ bmjopen-2019-036484

- Prepublication history and additional materials for this paper is available online. To view these files, please visit the journal online (http://dx.doi org/10.1136/bmjopen-2019036484).

Received 17 December 2019 Revised 02 June 2020 Accepted 25 July 2020

Check for updates

(C) Author(s) (or their employer(s)) 2020. Re-use permitted under CC BY-NC. No commercial re-use. See rights and permissions. Published by BMJ.

${ }^{1}$ Neonatal Intensive Care Unit, Children's Hospital 1, Ho Chi Minh City, Vietnam

${ }^{2}$ Department of Paediatric and Adolescence, Rigshospitalet, Copenhagen, Denmark ${ }^{3}$ Oxford University Clinical Research Unit, Ho Chi Minh City, Vietnam

${ }^{4}$ Nuffield Department of Medicine, University of Oxford, Oxford, United Kingdom

Correspondence to Dr Chuong Huu Thieu Do; thieuchuong@yahoo.com

\section{ABSTRACT}

Objectives To describe the characteristics of rehospitalisation in Vietnamese preterm infants and to examine the time-to-first-readmission between two gestational age (GA) groups (extremely/very preterm (EVP) vs moderate/late preterm (MLP)); and further to compare rehospitalisation rates according to GA and corrected age (CA), and to examine the association between potential risk factors and rehospitalisation rates.

Design and setting A cohort study to follow up preterm infants discharged from a neonatal intensive care unit (NICU) of a tertiary children's hospital in Vietnam.

Participants All preterm newborns admitted to the NICU from July 2013 to September 2014.

Main outcomes Rates, durations and causes of hospital admission during the first 2 years.

Results Of 294 preterm infants admitted to NICU (all outborn, GA ranged from 26 to 36 weeks), 255 were discharged alive, and 211 (83\%) NICU graduates were followed up at least once during the first 2 years CA, of whom $56 \%$ were hospital readmitted. The median (IQR) of hospital stay was $7(6-10)$ days. Respiratory diseases were the major cause (70\%). Compared with MLP infants, EVP infants had a higher risk of first rehospitalisation within the first 6 months of age $(p=0.01)$. However, the difference in risk declined thereafter and was similar from 20 months of age. There was an interaction in rehospitalisation rates between GA and CA. Longer duration of neonatal respiratory support and having older siblings were associated with higher rehospitalisation rates. Lower rates of rehospitalisation were seen in infants with higher cognitive and motor scores (not statistically significant in cognitive scores).

Conclusions Hospital readmission of Vietnamese preterm infants discharged from NICU was frequent during their first 2 years, mainly due to respiratory diseases. Scale-up of follow-up programmes for preterm infants is needed in low-income and middle-income countries and attempts to prevent respiratory diseases should be considered.

\section{INTRODUCTION}

Preterm infants (born before 37 completed weeks of gestation) are at high risk of morbidities immediately after birth and during childhood. ${ }^{12}$ While long-term neurodevelopment,
Strengths and limitations of this study

- The cohort represents one of the largest and longest follow-ups of preterm infants in Vietnam and similar settings.

- This study addressed several aspects of hospital readmission of Vietnamese preterm infants with a wide range of gestational age.

- A single centre-based study may limit the generalisation of findings to the broader population of preterm infants.

- The criteria for hospital admission may be varied among paediatricians and healthcare centres.

- The International Classification of Diseases code assignments may be varied among paediatricians.

especially disability and handicap, is of major concern and thoroughly studied, short-term morbidities in infancy draw less attention and are not widely understood. Studies in high-income countries have demonstrated that preterm infants are more prone to morbidities requiring hospitalisation in early childhood..$^{3-5}$ Data in low-income and middleincome countries (LMICs) are of major interest as an increasing number of preterm infants now survive and are discharged from neonatal intensive care.

Rates and causes of hospitalisation are indicators of major child morbidities. The rate of hospitalisation among preterm infants was reported to be highest in the first years of life and decreased over time. ${ }^{6}$ Preterm infants with a lower gestational age (GA) are at a higher risk, possibly due to a higher rate of respiratory problems, for example, chronic lung disease (CLD). ${ }^{6-9}$ Other neonatal morbidities including brain injuries (intraventricular haemorrhage or periventricular leucomalacia), retinopathy of prematurity (ROP) and necrotising enterocolitis (NEC) are also known risk factors for later hospital 
readmission in the first years of life. ${ }^{10}$ The susceptibility of preterm infants is possibly attributed to the early injuries of immature organs and relative immunodeficiency. ${ }^{11}$ Further, the influence of deprived living conditions such as suboptimal nutritional status, high prevalence of infectious diseases and contaminated environments could result in even higher morbidity rates among preterm infants in LMICs. ${ }^{12}$

The South of Vietnam consists of 32 provinces and cities with a population of approximately 50.1 million, half of the country's population, and Ho Chi Minh City is the largest city. Neonatal care in the public health sector is free of charge through the social insurance system and is provided by a system of maternity hospitals, children hospitals, tertiary hospitals and primary care clinics. There are two major maternity hospitals (Tu Du Hospital and Hung Vuong Hospital), with approximately 13000 preterm births in a total of 110000 births annually. The two major children hospitals without obstetric care (Children's Hospital 1 and Children's Hospital 2) receive approximately 11000 newborns annually from all healthcare facilities in the catchment area covering the South of Vietnam. Among these newborns, about 700 preterm newborns are admitted to the neonatal intensive care unit (NICU) (annual reports and personal communication, 2018). For preterm newborns with birth weight less than $1000 \mathrm{~g}$, the need for resuscitation at delivery room and NICU referral is discussed in the consultation with the parents on a case-by-case basis.

From a public health perspective, increased child morbidity following the rising number of preterm survivors could impose substantial constraints on the healthcare system. ${ }^{13}$ The burden of perinatal care for preterm births has been investigated extensively. ${ }^{14-16}$ However, to our knowledge, the morbidities of these infants after discharge from the NICU in Vietnam and similar LMICs have not been well documented. ${ }^{17}$

In this exploratory study, we estimated the rehospitalisation rates and assessed the association between these rates and several risk factors, including neurodevelopmental scores, for Vietnamese preterm infants in the first 2 years of life. First, we estimated and compared the rehospitalisation rates according to GA and corrected age (CA). Further, we compared the time-to-first-readmission between the two GA groups, extremely/very preterm (EVP) versus moderate/late preterm (MLP) infants. Second, we described the total numbers, durations and causes of rehospitalisation according to the GA groups (EVP and MLP). Third, we examined the association between neonatal and demographic factors, plus neurodevelopmental scores, and rates of rehospitalisation.

\section{METHODS}

The cohort study was conducted at the NICU at Children's Hospital 1, Ho Chi Minh City, Vietnam. The study hospital is one of two major tertiary centres responsible for neonatal intensive care in the South of Vietnam. The unit has 30 beds and 1200 admissions annually, of which the majority are transferred from healthcare facilities providing obstetrical care and a few are directly admitted to the emergency room from home. Written parental consent was obtained.

All preterm newborns admitted to the unit from July 2013 to September 2014 were eligible for enrolment if they fulfilled the criteria: $<37$ completed weeks of gestation at birth, age at admission <29 days, and no congenital brain malformation or chromosome anomaly. Newborns admitted to NICU less than 72 hours only, or for treatment of ROP, were excluded. Gestational age was defined as completed weeks of gestation and was determined using one of the three following methods in order of priority: antenatal ultrasound before 20 weeks of gestation, the last menstrual day or clinical examination using the New Ballard Score ${ }^{18}$ Enrolled newborns were classified by GA, including EVP (GA $<32$ completed weeks) and MLP (GA from 32 to 36 completed weeks). They were discharged when being able to oral feed and showing steadily appropriate weight gain and clinical stability for at least 3 days. Then they were followed up until 24 months CA, with the scheduled visits at 3,12 and 24 months. CA in months was calculated by subtracting the weeks remaining to complete 40 weeks from the chronological age. ${ }^{19}$ Demographic characteristics and neonatal morbidities were collected during neonatal hospitalisation and at follow-up visits (see online supplemental appendix).

A child health notebook was provided to the parents to record all child healthcare facility contacts, supplementing the review of medical events with the parents at follow-up visits. Follow-up was free of charge and transportation was provided if needed. The maximum distance to the study hospital among the participants was nearly 450 $\mathrm{km}$. Rehospitalisation was defined as hospital admission requiring at least one overnight stay in a hospital after first discharge from NICU. All hospital readmissions were recorded, regardless of which hospital. Data on rehospitalisation were collected from the notebook, discharge papers and interviews with caregivers. Data included age in months at hospital readmission and duration (in days) of hospital stay, main symptoms, primary diagnosis, and treatments. Repeated hospital admission within 2 days was considered as a single hospitalisation. Discharge papers given to the parents by the healthcare clinics were used to verify information in most cases. Only primary diagnosis was recorded for each hospital readmission. The main diagnosis was classified into one of five categories: respiratory diseases, digestive diseases, infectious diseases (other than respiratory and gastrointestinal infections), surgical intervention and others. Data on rehospitalisations of no-shows or deceased infants were obtained by telephone interviews with the parents but were finally excluded due to possible recall biases.

Further, at 24 months CA, neurodevelopment of the infants was assessed using subtests of Bayley Scales of Infant and Toddler Development-3rd Edition (Bayley-III), 
including cognitive, fine motor and gross motor scores (see online supplemental appendix). ${ }^{20}$

\section{Patient and public involvement}

The idea and development of this study were informed by the lack of standardised follow-up of preterm infants in Vietnam. In our clinical practice, families expressed the need for better understanding of child morbidity after discharge from neonatal intensive care, including risks of hospital readmission. Families were directly involved in data collection, as they monitored and documented their infant's health during the study period. Medical history and examination findings were shared at regular follow-up consultations to inform plans for the individual infant. Further, the overall study results and conclusions will be disseminated to the families having preterm infants at subsequent follow-up visits.

\section{Statistical analysis}

Demographic and clinical characteristics are summarised as mean (SD) for continuous variables and absolute count (\%) for categorical variables, except for the length of respiratory support and the length of neonatal hospital stay being presented as median (IQR) due to a skewed distribution.

Rehospitalisation rates were calculated as the number of hospital readmissions per month after the first neonatal discharge. The lengths of hospital stays are presented as median (IQR) in days. The total number of rehospitalisation, the number of infants having at least one rehospitalisation and the causes of hospital readmissions are presented as absolute count (\%). $\chi^{2}$ test for independence was used to identify the difference in rehospitalisation causes between the two GA groups, EVP versus MLP (hence we did not correct for repeated hospitalisations within an individual). The distribution of time-to-first-readmission was examined and illustrated by Kaplan-Meier curves according to the two GA groups. We tested for differences between the two GA groups using the log-rank test with follow-up time restricted to $3,6,12$ and 24 months to take account of non-proportionality in the hazard over ages. We used a Poisson regression model with generalised estimating equation to estimate the overall rate of rehospitalisation related to potential risk factors including gender, GA, need of mechanical ventilation, duration of respiratory support, neonatal morbidities (NEC, sepsis and surgery), maternal factors (age and educational level), having older siblings at home, and cognitive and motor Bayley-III scores. These candidate covariates were identified by a review of the literature on risk factors for morbidities in infancy. ${ }^{6} 10122122$ The Bayley scores were based on our hypothesis and were not included together, but one at a time due to a strong correlation $(\mathrm{r}=0.6)$ between them. We used generalised estimating equations to correct for repeated events

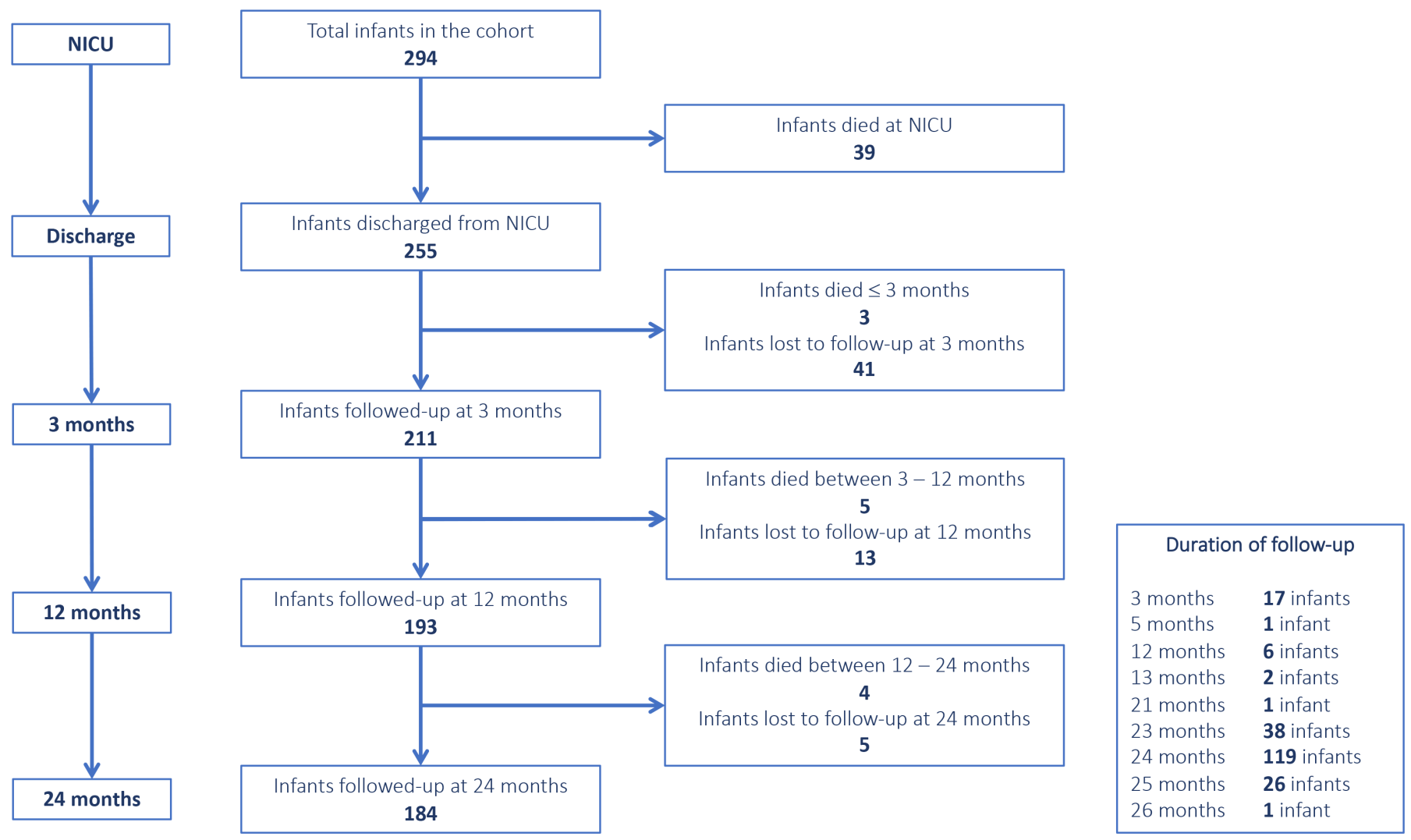

Figure 1 Flow chart of preterm infants from NICU discharge to 24-month follow-up. Actual follow-up time was less than 24 months in some patients. The reasons for attrition of 32 infants were listed as being unable to contact (11), travel problems (7), languages of minor ethnics (3), living abroad (3), well-being status (4) and unspecified (4). NICU, neonatal intensive care unit. 
Table 1 Demographic and clinical characteristics of Vietnamese preterm infants followed up during 2 years after NICU discharge

\begin{tabular}{|c|c|c|c|}
\hline \multirow[b]{3}{*}{ Characteristics } & \multicolumn{3}{|c|}{$\mathrm{n}(\%)$ or mean (SD) or median (IQR) } \\
\hline & \multicolumn{2}{|l|}{ Gestational age (GA) } & \multirow{2}{*}{$\begin{array}{l}\text { Total } \\
\text { (N=211) }\end{array}$} \\
\hline & GA $<32$ weeks $(n=100)$ & $G A \geq 32$ weeks $(n=111)$ & \\
\hline Boys, n (\%) & $62(62)$ & $72(65)$ & $134(64)$ \\
\hline Birth weight (g), mean (SD) & $1410(258)$ & $2088(388)$ & $1767(475)$ \\
\hline GA (weeks), mean (SD) & $29.6(1.4)$ & $33.6(1.4)$ & $31.6(2.4)$ \\
\hline \multicolumn{4}{|l|}{ GA distribution (weeks), n (\%) } \\
\hline$<28$ & $12(12)$ & - & $12(6)$ \\
\hline $28-32$ & $88(88)$ & - & $88(42)$ \\
\hline $32-34$ & - & $62(56)$ & $62(29)$ \\
\hline $34-37$ & - & $49(44)$ & $49(23)$ \\
\hline Multiple births (all twins), n (\%) & $17(17)$ & $17(15)$ & $34(16)$ \\
\hline Mother's age at birth (year), mean (SD) & $28.7(5.8)$ & $28.8(6.1)$ & $28.8(5.9)$ \\
\hline \multicolumn{4}{|l|}{ Maternal education*, n (\%) } \\
\hline Junior high school or less ( $\leq 9$ years) & $62(62)$ & $76(68)$ & $138(65)$ \\
\hline Senior high school or higher (>9 years) & $38(38)$ & $35(32)$ & $73(35)$ \\
\hline \multicolumn{4}{|l|}{ Maternal occupation, n (\%) } \\
\hline Skilled job† & $19(19)$ & $19(17)$ & $38(18)$ \\
\hline Housewife & $31(31)$ & $30(27)$ & $61(29)$ \\
\hline Farmer & $15(15)$ & $11(10)$ & $26(12)$ \\
\hline Others $\ddagger$ & $35(35)$ & $51(46)$ & $86(41)$ \\
\hline Primary care by parents§, $\mathrm{n}(\%)$ & $90(90)$ & $101(91)$ & $191(91)$ \\
\hline Older siblings at home, $\mathrm{n}(\%)$ & $55(55)$ & $66(59)$ & $121(57)$ \\
\hline Ethnic minority (non-Kinh)ף, n (\%) & $1(1)$ & $3(3)$ & $4(2)$ \\
\hline Major birth defects, n (\%) & $4 / 100(4)$ & $23 / 110(21)$ & $27 / 210(13)$ \\
\hline Mechanical ventilation, $\mathrm{n}(\%)$ & $53 / 100(53)$ & $53 / 110(48)$ & $106 / 210(50)$ \\
\hline Length of respiratory support (day), median (IQR) & $22(12-44)$ & $7(4-12)$ & $11(6-24)$ \\
\hline Chronic lung disease, $n(\%)$ & $32 / 99$ (32) & $6 / 110(5)$ & $38 / 209(18)$ \\
\hline With postnatal corticosteroids, n (\%) & $7(22)$ & $2(33)$ & $9(24)$ \\
\hline Without postnatal corticosteroids, n (\%) & $25(78)$ & $4(67)$ & $29(76)$ \\
\hline Sepsis, n (\%) & $78 / 99(79)$ & $62 / 110(56)$ & $140 / 209(67)$ \\
\hline Positive blood culture & $12(15)$ & $14(23)$ & $26(19)$ \\
\hline Negative blood culture ${ }^{\star *}$ & $66(85)$ & $48(77)$ & $114(81)$ \\
\hline Necrotising enterocolitis, n (\%) & $11 / 99(11)$ & 3/110 (3) & $14 / 209(7)$ \\
\hline Surgery††, n (\%) & 13/100 (13) & $23 / 110(21)$ & $36 / 210(17)$ \\
\hline Laser ROP, n (\%) & $15 / 96(16)$ & $1 / 56(2)$ & $16 / 152(11)$ \\
\hline Length of stay (days), median (IQR) & $48(36-69)$ & $22(15-34)$ & $34(19-50)$ \\
\hline
\end{tabular}

Data on income status were not available due to indeterminate responses from parents' perspective.

Missing data: one for mother's age at birth and two for length of respiratory support.

Because of rounding, percentages may not total 100.

${ }^{*}$ The first 9 years includes 5 years in elementary school and 4 years in junior high school. Higher educational level includes 3 years in senior high school, college, undergraduate and postgraduate education.

†Skilled job refers to professional and intellectual work.

¥Other jobs refer to unskilled labour and shopkeeper.

$\S$ Other primary caregivers were grandparents or other relatives.

ףEthnic minority includes Khmer and Chinese.

${ }^{*}$ Suspected sepsis based on clinical signs and biomarkers for septicaemia.

††Surgery includes repairs of congenital malformations (26), volvulus from intestinal malrotation (1), peritonitis due to gastrointestinal perforation (5) and patent ductus arteriosus ligation (4).

$\mathrm{NICU}$, neonatal intensive care unit; ROP, retinopathy of prematurity. 
within individuals. The duration of respiratory support was log-transformed with base 2 to make its distribution less skewed (see online supplemental appendix). We modelled GA and follow-up time (ie, CA) via restricted cubic splines and included an interaction term between both variables. We tested whether the interaction term and the non-linear term improved model fit. Finally, we excluded the non-linear components of the interaction between GA and CA $(\mathrm{p}=0.35)$, and only used a linear interaction term $(\mathrm{p}=0.007)$ in the model.

All statistical analyses were performed using R V.3.5.0 software.

\section{RESULTS}

Of 294 preterm infants (all outborn) enrolled during the study period, 255 were discharged alive, and 211 (83\%) NICU graduates were followed up at least once in the first 2 years (figure 1). Among these infants, GA ranged from 26 to 36 completed weeks. The mean (SD) birth weight and GA of follow-up infants were 1767 (475) $\mathrm{g}$ and 31.6 (2.4) weeks compared with birth weight of 1877 (473) g and GA of 32.7 (2.4) weeks of 32 out of 255 (13\%) infants lost to follow-up. None received oxygen therapy at home after discharge.

Table 1 presents the demographic and clinical characteristics of the studied infants by GA groups (EVP and MLP). The demographic characteristics were similar between the two groups. Of the clinical characteristics at discharge after birth, MLP infants had higher birth weight and higher percentage of major birth defects and thus neonatal surgery, whereas EVP infants had longer respiratory support, longer neonatal hospital stay, and higher percentages of morbidities including CLD, NEC, ROP and sepsis. Of note, the percentages of infants in need of mechanical ventilation were similar between the two groups (see online supplemental appendix table A).

Figure 2 depicts the Kaplan-Meier curves for the timeto-first-readmission according to GA groups (EVP vs MLP). In comparison with MLP infants, EVP infants had initially a higher risk of hospital readmission ( $p$ value logrank test until 3 months 0.004 and until 6 months 0.01 ), but the risk difference decreased over time and the estimates coincide after 20 months ( $\mathrm{p}$ value log-rank tests until 12 months and until 24 months 0.2).

Table 2 shows the numbers, durations and causes of rehospitalisation during the first 2 years of life. A total of 231 events were recorded for 211 preterm infants in the cohort, equivalent to a follow-up of 4581 patient-months.

Among 193 infants who completed 1-year follow-up, 90 $(47 \%)$ were readmitted at least once in the first year. Of 184 infants who completed 2-year follow-up, 41 (22\%) were readmitted at least once in the second year and 81 $(44 \%)$ were not hospitalised throughout the first 2 years. Specifically, two infants were readmitted 10 and 13 times throughout the first 2 years. The median (IQR) hospital stay was 7 (6-10) days, with the longest stay being 91 days. Respiratory diseases accounted for $70 \%$ of all causes $(74 \%$ in the first year and $60 \%$ in the second year), followed by other infectious diseases (15\%) and digestive diseases $(9 \%)$. A small percentage of rehospitalisations were due to surgery and other causes (6\%). The causes of rehospitalisation between EVP and MLP infants were mostly the same $(\mathrm{p}=0.2)$.

Figures 3 and 4 present the rates of rehospitalisation per month according to represented GAs (27, 31 and 35 weeks GA). It shows the interaction of rehospitalisation rates between GA and CA. Specifically, while the CI showed large overlaps and the rehospitalisation rates
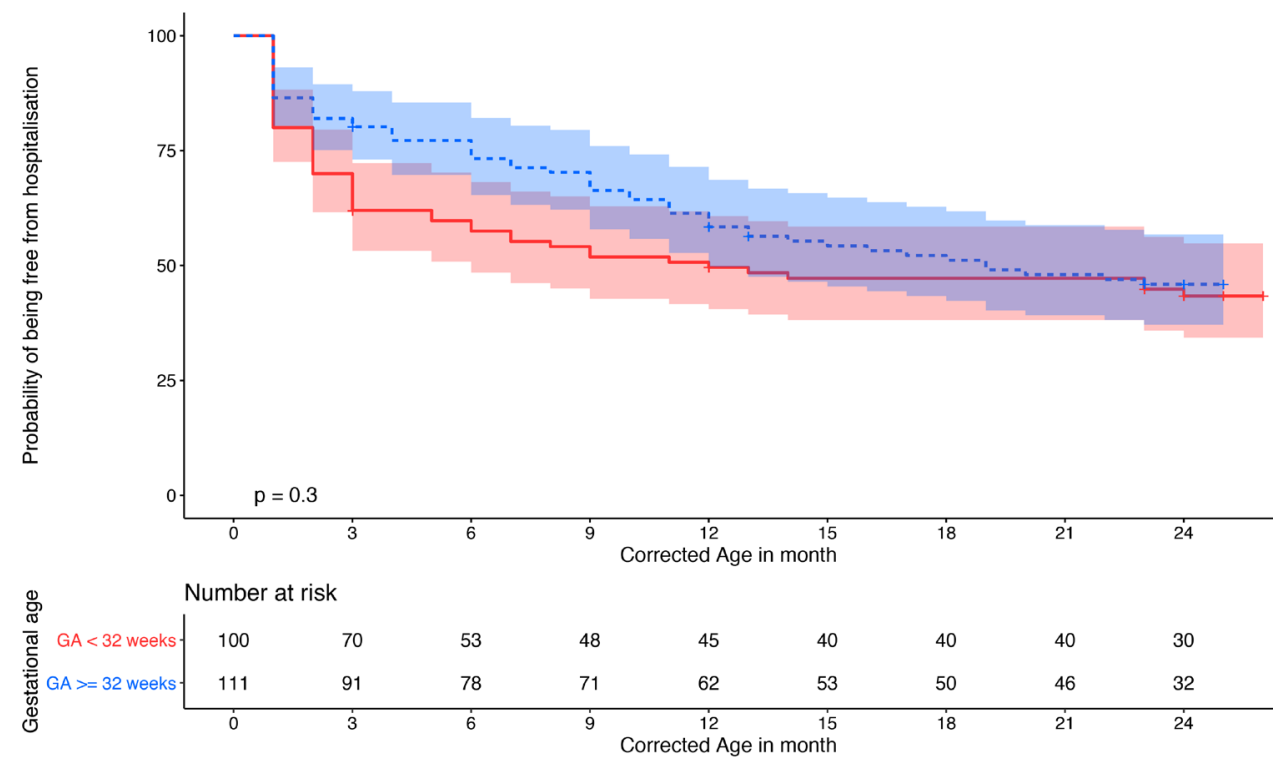

Gestational age $+\mathrm{GA}<32$ weeks $+-\mathrm{GA}>=32$ weeks

Figure 2 Kaplan-Meier curves for the time-to-first-readmission according to two gestational age (GA) groups. The 95\% Cls are illustrated by the shaded areas in the corresponding colours. 
Table 2 Numbers, durations and causes of rehospitalisation of Vietnamese preterm infants during the first 2 years of life

\begin{tabular}{|c|c|c|c|}
\hline & \multicolumn{2}{|c|}{ n (\%) or median (IQR) } & \multirow[b]{3}{*}{ Total $(\mathrm{N}=\mathbf{2 1 1})$} \\
\hline & \multicolumn{2}{|c|}{ Gestational age (GA) } & \\
\hline & $\begin{array}{l}\text { GA <32 weeks } \\
(n=100)\end{array}$ & $\begin{array}{l}\mathrm{GA} \geq 32 \text { weeks } \\
(n=111)\end{array}$ & \\
\hline Rehospitalisation, n (\%) & 109 & 122 & 231 \\
\hline 0-6 months, $\mathrm{n}(\%)$ & $59(54)$ & $43(35)$ & $102(44)$ \\
\hline 6-12 months, $n(\%)$ & $31(28)$ & $30(25)$ & $61(26)$ \\
\hline 12-24 months, $\mathrm{n}(\%)$ & $19(17)$ & $49(40)$ & $68(29)$ \\
\hline Number of infants having at least one rehospitalisation, $\mathrm{n}(\%) \dagger$ & $49 / 86(57)$ & $54 / 98(55)$ & $103 / 184(56)$ \\
\hline Only once & $27(55)$ & $26(48)$ & $53(51)$ \\
\hline Twice & $9(18)$ & $15(28)$ & $24(23)$ \\
\hline$\geq 3$ times & $13(27)$ & $13(24)$ & $26(25)$ \\
\hline Causes of rehospitalisation, $\mathrm{n}(\%) \ddagger$ & & & $n=231$ \\
\hline Respiratory diseases & $78(72)$ & $83(68)$ & $161(70)$ \\
\hline Infectious diseases & $13(12)$ & $21(17)$ & $34(15)$ \\
\hline Digestive diseases & $10(9)$ & $11(9)$ & $21(9)$ \\
\hline Surgical diseases & $8(7)$ & $4(3)$ & $12(5)$ \\
\hline Others & $0(0)$ & $3(2)$ & $3(1)$ \\
\hline Duration of rehospitalisation (days), median (IQR) & $7(6-11)$ & $7(6-9)$ & $7(6-10)$ \\
\hline
\end{tabular}

Because of rounding, percentages may not total 100 .

*Among 211 infants, 193 infants completed 1-year follow-up and 184 infants completed 2-year follow-up.

†Data from 184 infants completed 2-year follow-up.

$\ddagger$ Causes of rehospitalisation: respiratory diseases (apnoea, bronchiolitis, pneumonia, croup, suspected asthma), infectious diseases (other than respiratory and gastrointestinal diseases including viral infection (Dengue, hand-foot-mouth, unspecified), neonatal infection, meningitis, urinary tract infection), digestive diseases (diarrhoea, enterocolitis, gastro-oesophageal reflux, conjugated hyperbilirubinaemia), surgical diseases (retinopathy of prematurity, closure of stoma (ileostomy or colostomy), inguinal hernia repair, cleft lips closure, ventricular septal defect closure), and others (cyanotic spells, febrile convulsion).

could be similar among GAs in the first months after NICU discharge, the point estimates of rates were higher for those with lower GA. Following that, these rates of infants with lower GA reduced markedly over time, whereas these rates of infants with higher GA reduced moderately. In the second year, these rates became positively associated with GA (see online supplemental appendix table B).

Table 3 presents unadjusted and adjusted rate ratios and $95 \%$ CI of various factors for hospital readmission. We found a clear relation between GA and the rate of readmission (overall $\mathrm{p}$ value for $\mathrm{GA}=0.007$ ), as well as between current age and readmission rate (overall $\mathrm{p}$ value for $\mathrm{CA}<0.001$ ). To quantify the relation between GA and CA and rate of readmission, taking account of the interaction between GA and CA, we report the rate ratio for GA 35 vs 27 at two values of CA as well as the rate ratio of CA for two values of GA. Girls were less likely to be rehospitalised compared with boys in the first 2 years, but it was not statistically significant (RR $0.76,95 \% \mathrm{CI}$ 0.51 to $1.12, \mathrm{p}=0.16)$. The duration of neonatal respiratory support and older siblings at home increased the risk of rehospitalisation both in univariate analysis and in multivariable analysis. Specifically, when the duration of neonatal respiratory support doubled, the rate of rehospitalisation increased by $26 \%$ (RR 1.26, 95\% CI 1.08 to 1.48 , $\mathrm{p}=0.004)$. Living with older siblings at home increased rehospitalisation rates by $69 \%$ (RR $1.69,95 \%$ CI 1.12 to 2.56, $\mathrm{p}=0.013$ ). Infants with higher cognitive and motor Bayley-III scores were less likely to be rehospitalised. Each 15-point (1 SD) increase in cognitive and motor scores resulted in a decrease of $26 \%$ (RR $0.74,95 \%$ CI 0.53 to $1.05, \mathrm{p}=0.092$ ) and $29 \%$ (RR $0.71,95 \%$ CI 0.54 to 0.94 , $\mathrm{p}=0.015$ ) in the point estimates of rehospitalisation rates (see online supplemental appendix table $\mathrm{C}$ ).

\section{DISCUSSION}

In this exploratory study of rehospitalisation of Vietnamese preterm infants discharged from the NICU, we found that the majority of these infants $(56 \%)$ were readmitted to hospitals during the first 2 years $(47 \%$ in the first year and $22 \%$ in the second year). Respiratory diseases were the major cause (70\%). Compared with MLP infants, EVP infants had a significantly higher risk of first hospital readmission within the first 6 months of age. However, the difference in risk declined thereafter and became similar from 20 months. Among known risk factors, longer duration of neonatal respiratory support and having older siblings at home were associated with higher rates of rehospitalisation within the first 2 years. Further, higher 
Incidence of hospitalisation



Figure 3 Rehospitalisation rates of represented gestational age during the first 2 years. Data are derived from 211 Vietnamese preterm infants with gestational ages ranging from 26 to 36 weeks. Rehospitalisation rates are estimated using a Poisson regression model using generalised estimating equations and are illustrated by solid lines with $95 \% \mathrm{Cls}$ as shaded areas. The selected gestational ages of 27, 31 and 35 weeks are shown in red, blue and green, respectively.

cognitive and motor Bayley-III scores showed a tendency towards lower rates of rehospitalisation.

The high risk of rehospitalisation (at least once) among our preterm infants is similar to some studies in highincome countries, ${ }^{23-27}$ but is much higher than those among preterm published by Underwood et a ${ }^{28}(15 \%$ in the first year) and by Laugier $e t a l^{12}$ (22\% in the first year). Our preterm infants were three times more likely to be rehospitalised in the first year compared with only $10 \%-15 \%$ of full-term infants reported by Slimings et al. ${ }^{6}$ This higher risk could be due to prematurity and severe neonatal condition among our study infants. Further, preterm infants may be viewed as vulnerable children by healthcare providers; thus, lower thresholds for hospital admission are probably common practice. Of note, $17 \%$ of infants were readmitted within the first month following NICU discharge, which is much higher than those found in full-term infants $(1.8 \%-3.7 \%) .{ }^{6}{ }^{29}$ Such high rates, however, show a similar trend from previous studies. ${ }^{30}$ On the one hand, this notifies paediatricians and caregivers of a high probability of morbidities within the first months caring at home. On the other hand, this raises concerns about discharge planning as well as a broad indication for hospital readmission.
Interestingly, we found an interaction between GA and CA in terms of rehospitalisation rates per month, whereas several studies reported GA inversely associated with rehospitalisation rates until school age or adolescence. ${ }^{3682831}$ In our study, infants with lower GA experienced higher rehospitalisation rates only in the first few months. Thereafter, the relation reversed, with higher rates found in infants with higher GA. This could be scrutinised by reference to the criteria of NICU admission. While EVP newborn is a criterion per se, newborns with higher GA are not admitted to our NICU unless in severe conditions and in need of intensive care, including mechanical ventilation. The gradual stability together with the steady improvement of health status has been seen among EVP infants over time. At the same time, the early injury to the lung due to severe neonatal morbidities and invasive intervention could result in enduring functional respiratory disorders. Specifically, two infants with the highest rehospitalisation events (10 and 13 times) were both 36 weeks GA, in need of mechanical ventilation, having prolonged respiratory support in NICU, and 21 of their total 23 hospital admissions were due to respiratory diseases. The synergic effect of prematurity and neonatal interventions on long-term outcomes 
Incidence of hospitalisation

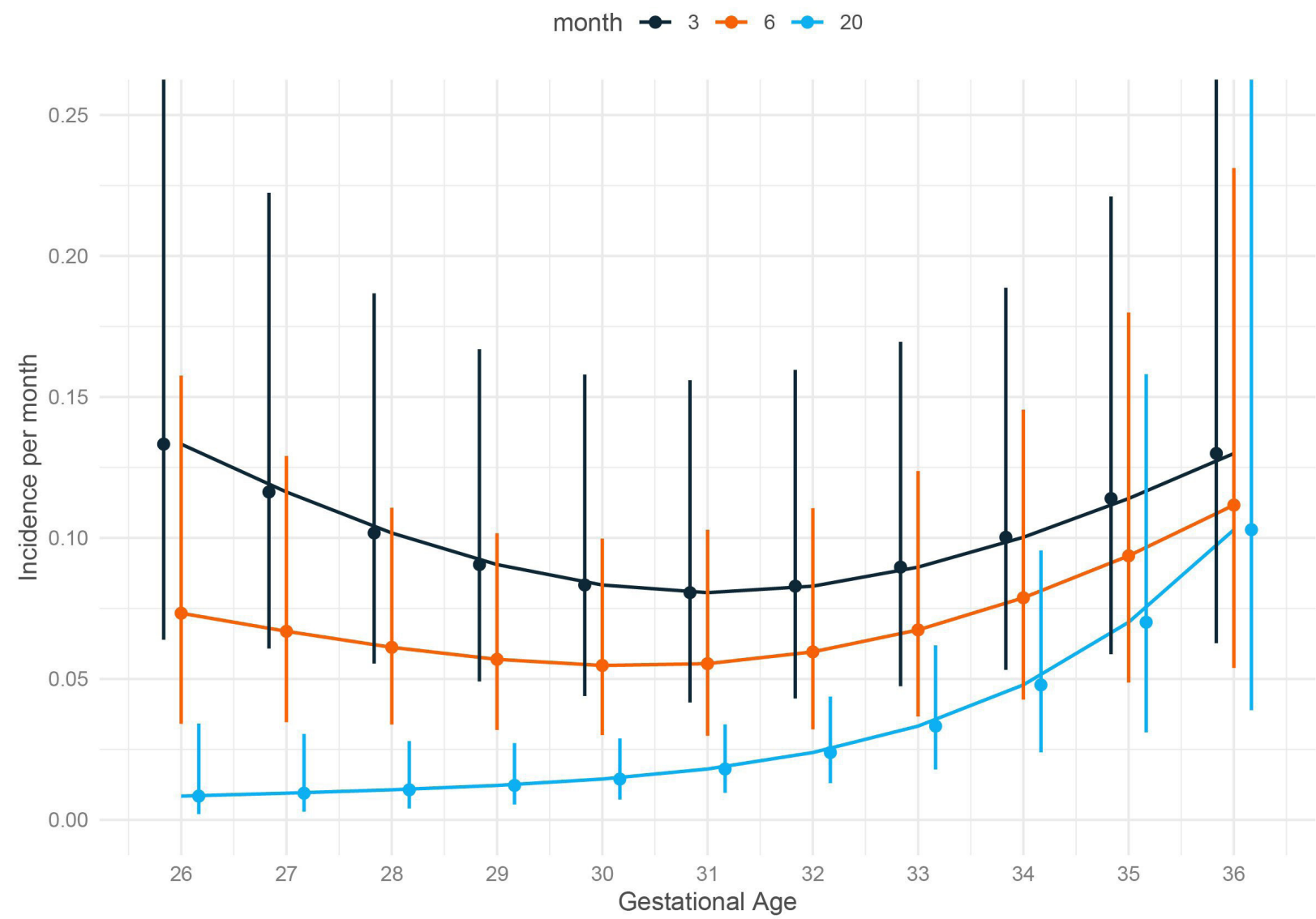

Figure 4 Rehospitalisation rates of represented corrected age during the first 2 years. Data are derived from 211 Vietnamese preterm infants with gestational ages ranging from 26 to 36 weeks. Rehospitalisation rates are estimated using a Poisson regression model using generalised estimating equations and are illustrated by solid lines with $95 \%$ Cls as vertical bars. The selected corrected ages of 3, 6 and 20 months are shown in black, orange and blue, respectively.

could explain this interaction and needs to be explored in further work.

Boys are at higher risk of rehospitalisation compared with girls in several reports, ${ }^{6} 912212732$ and we found a similar result. This could be attributable to biological vulnerability or cultural trait of Confucianism in Vietnamese families, which entitles boys to get more privilege than girls. We did not find the differences in rates of rehospitalisation among infants by maternal age and education, similar to a report by Ralser $e t a l^{27}$ but inconsistent with others. ${ }^{10} 12$ On-hand access to healthcare services and free of charge for infants less than 6 years old in Vietnam could lessen the impact of these factors. Meanwhile, living with older siblings at home was associated with higher rehospitalisation rates in our study. Older siblings in contact with pathogens in day-care centres or schools may cause an increased risk of exposure for the younger child at home. ${ }^{22}$ Importantly, we demonstrated that mechanical ventilation and prolonged respiratory support were associated with higher rehospitalisation rates similar to previous reports. ${ }^{21}{ }^{23}$ This may be plausible in the context of a high proportion of respiratory diseases. Besides, we found an inverse correlation between cognitive and motor Bayley-III scores at 2 years and rehospitalisation rates. This could indicate the higher susceptibility of the child with poorer neurodevelopment to major morbidities in the early stage of life. However, the literature is sparse, and whether this finding is a causal relationship needs to be clarified in future studies.

Respiratory illness is the major cause of rehospitalisation in our cohort, which is in line with most of the previous studies. ${ }^{7812242831}$ However, this high percentage of respiratory cause $(70 \%)$ among other causes is noticeable and is higher than most studies previously reported (from $40 \%$ to $50 \%$ ). ${ }^{12} 2733$ Pneumonia and other respiratory diseases remain the leading cause of childhood morbidity and are among the most common reasons for hospital admission in LMICs. ${ }^{34}$ Similarly, studies on Vietnamese infants found that acute respiratory infection remains the leading cause of childhood morbidity and mortality ${ }^{35-37}$ Nearly $10 \%$ of Vietnamese infants are hospitalised in the first 6 months due to pneumonia. ${ }^{35}$ Bhutta $e t a l^{8}$ suggested that undernutrition, zinc deficiency and lack of exclusive breast feeding are risk factors for pneumonia, together with crowding and air pollution, which are common in urban areas of Vietnam. As a preventive measure, WHO and UNICEF recommend that breast feeding, nutrition, vaccination and WASH (water, 
Table 3 Rate ratios of risk factors for rehospitalisation of Vietnamese preterm infants in the first 2 years of life

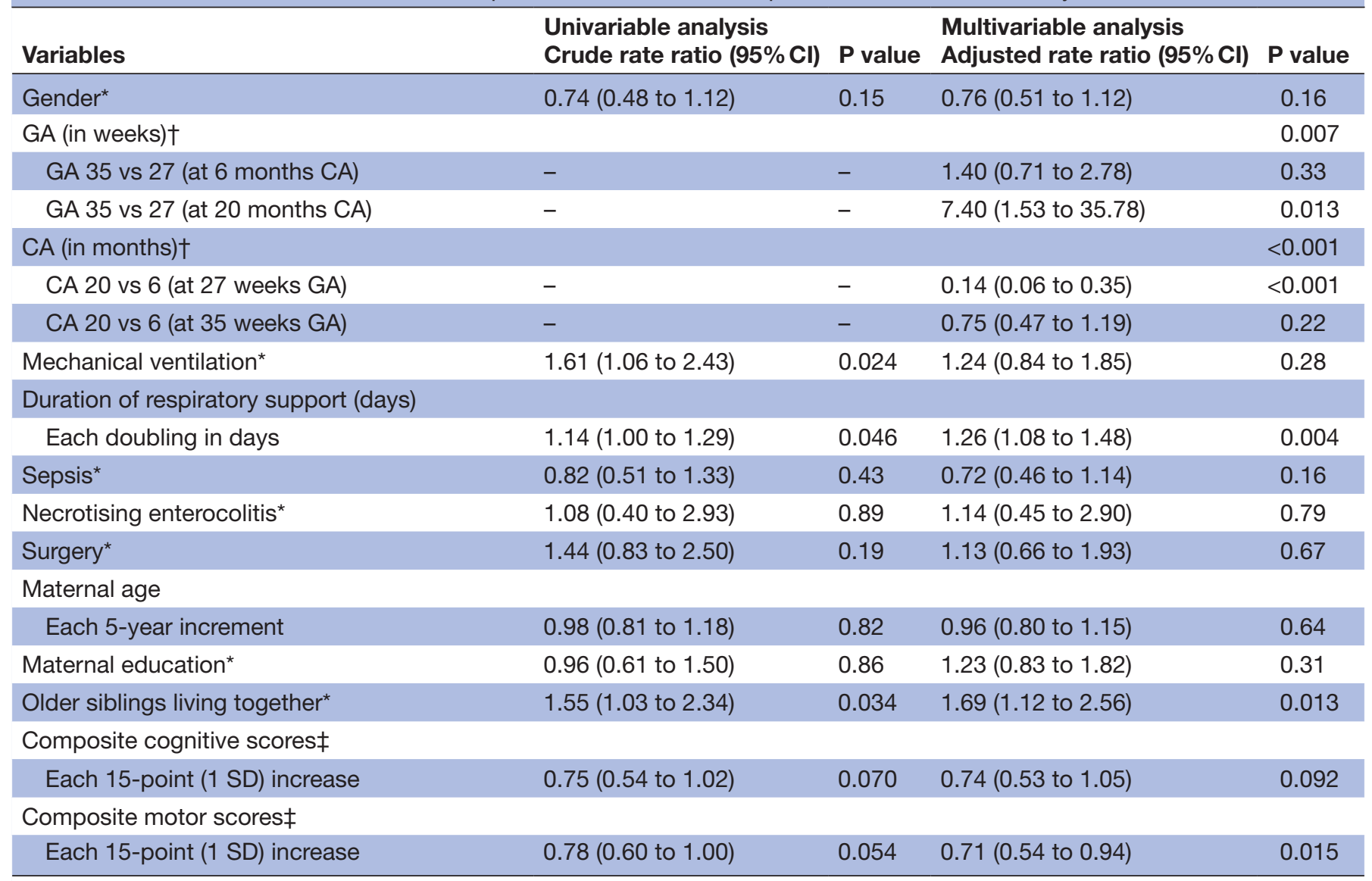

${ }^{*}$ Rate ratios were estimated as follows: for gender, girls relative to boys; for mechanical ventilation, sepsis, necrotising enterocolitis, surgery, older siblings living together, yes relative to no; for maternal education, higher level relative to lower level.

†Interactions between GA and CA were significant in the multivariable analysis with linear interaction term and cubic splines for main effects of $\mathrm{GA}$ and $\mathrm{CA}$.

$\ddagger$ Due to a strong correlation of cognitive and motor scores $(r=0.6)$, we added each score at a time in the multivariable model to obtain estimates and $\mathrm{Cl}$.

$\mathrm{CA}$, corrected age; GA, gestational age.

sanitation and hygiene) should become common practices. Noticeably, half of our infants required mechanical ventilation and one in every five required prolonged respiratory support. These factors could superimpose to the innately immature lung and increase vulnerability to later respiratory infection, partly explained for such a high percentage of respiratory diseases.

\section{Strengths and limitations}

The prospective design is one of the strengths of our study and the cohort represents one of the largest and longest follow-up groups of preterm infants in Vietnam and similar LMICs. We conducted a study on several aspects of hospital readmission across the full spectrum of GA using currently recommended preterm definitions.

Nevertheless, we acknowledge several limitations, including the lack of robustness inherent in a single centre-based study and a cohort with the most severe outborn newborns in the South of Vietnam. These factors may limit the representativeness of our findings to the broader population of children born prematurely. In this exploratory study, our approach was pragmatic. The small sample size of 211 infants could limit the power of our statistical analyses. The number of infants who were lost to follow-up or died in the 2-year period accounted for $17 \%$ (44 of 255) of NICU graduates. This potentially results in selection bias due to probably more severe illness in dead infants or less readiness to hospital visits in the group lost to follow-up. Risk factors of hospital admission including socioeconomic status, living conditions and other risks of respiratory infection (parental smoking and day-care attendance) were not available in our cohort. The indications for hospital admission were varied among healthcare facilities, although Integrated Management of Childhood Illness practice has been disseminated in the region. Further, there is no linked system to record admissions or discharges between hospitals in the South of Vietnam; therefore, data had to be collected from discharge papers and by interviews with caregivers, which was potentially prone to recall bias. Although we have taken measures to reduce spurious information, including check-recheck 
queries, to what extent this bias affects our results is unknown. The practice of International Classification of Diseases-10th coding is not standardised and there may be some variability in the assignment of the primary diagnosis, but this type of error could be lessened by grouping diagnoses into broader categories. Finally, we did not take secondary diagnosis into account, which may have underestimated the impact of secondary issues.

\section{CONCLUSION}

In this study, Vietnamese preterm infants discharged from NICU experienced a high rate of hospital readmission during the first 2 years of life, with respiratory diseases being the most common cause. Prolonged neonatal respiratory support and living with older siblings were associated with higher rehospitalisation rates. Further, neurodevelopmental scores were also potential indicators showing an inverse relation. Scale-up of standard follow-up programmes of preterm infants in LMICs is needed, especially after NICU discharge. It could be considered to include information to parents about the likelihood of emerging illnesses during the first few months after discharge and possible preventive practices should be explained, especially in relation to respiratory diseases. These could improve the health of preterm infants and reduce the rates of hospital admission.

Acknowledgements The authors are grateful to the Bayley team of Oxford University Clinical Research Unit (OUCRU) including Pham Ngoc Thanh, Saraswathy Sabanathan, Nguyen Thi Xuan Hoa, Pham Tam Dinh, Nguyen Thi Ngoc Nhi, Vo Ngoc Cat Tuong, Nguyen Thi Kim Anh and To Thi Mai Dao for their contribution to Bayley-III evaluations. The authors are also grateful to the staff of Children's Hospital 1 for their contribution to the administrative and coordinating work. The authors are also thankful to Bridget Wills, Saraswathy Sabanathan, the Laerdal Foundation, the Augustinus Foundation, Wellcome Trust and DANIDA for supporting funding and the Bayley-III tool. The authors wish to thank all families and infants who kindly participated in the project.

Contributors This study was conceptualised by CHTD, FKP, AYK and MLB. Data collection and management were performed by CHTD. Data were analysed by RBG, CHTD and MLB. CHTD, AYK, MLB and RBG wrote the manuscript with input from all authors. All authors read and approved the final version of the manuscript.

Funding The study was funded by the Laerdal Foundation (grant number 3452), the Augustinus Foundation (grant number 18-3731) and DANIDA, Denmark. RBG was supported by the Wellcome Trust (grant number 106680/B/14/Z).

\section{Competing interests None declared.}

Patient and public involvement Patients and/or the public were involved in the design, or conduct, or reporting, or dissemination plans of this research. Refer to the Methods section for further details.

Patient consent for publication Parental/guardian consent obtained.

Ethics approval The study was approved by the institutional review board of the study hospital.

Provenance and peer review Not commissioned; externally peer reviewed.

Data availability statement Data are available in a public, open access repository. Extra data can be accessed via the Dryad Data Repository at http://datadryad.org/ with doi:10.5061/dryad.dz08kprt9.

Open access This is an open access article distributed in accordance with the Creative Commons Attribution Non Commercial (CC BY-NC 4.0) license, which permits others to distribute, remix, adapt, build upon this work non-commercially, and license their derivative works on different terms, provided the original work is properly cited, appropriate credit is given, any changes made indicated, and the use is non-commercial. See: http://creativecommons.org/licenses/by-nc/4.0/.

\section{ORCID iDs}

Chuong Huu Thieu Do http://orcid.org/0000-0002-0461-059X

Ronald Bertus Geskus http://orcid.org/0000-0002-2740-3155

\section{REFERENCES}

1 WHO. Born too soon: the global action report on preterm birth, 2012.

2 Luu TM, Rehman Mian MO, Nuyt AM. Long-term impact of preterm birth: neurodevelopmental and physical health outcomes. Clin Perinatol 2017;44:305-14.

3 Boyle EM, Poulsen G, Field DJ, et al. Effects of gestational age at birth on health outcomes at 3 and 5 years of age: population based cohort study. BMJ 2012;344:e896.

4 Paranjothy S, Dunstan F, Watkins WJ, et al. Gestational age, birth weight, and risk of respiratory hospital admission in childhood. Pediatrics 2013;132:e1562-9.

5 Srinivasjois R, Slimings C, Einarsdóttir K, et al. Association of gestational age at birth with reasons for subsequent hospitalisation: 18 years of follow-up in a Western Australian population study. PLoS One 2015;10:e0130535.

6 Slimings C, Einarsdóttir K, Srinivasjois R, et al. Hospital admissions and gestational age at birth: 18 years of follow up in Western Australia. Paediatr Perinat Epidemiol 2014;28:536-44.

7 Houweling LMA, Bezemer ID, Penning-van Beest FJA, et al. First year of life medication use and hospital admission rates: premature compared with term infants. J Pediatr 2013;163:61-6.

8 Ray KN, Lorch SA. Hospitalization of early preterm, late preterm, and term infants during the first year of life by gestational age. Hosp Pediatr 2013;3:194-203.

9 Kuzniewicz MW, Parker S-J, Schnake-Mahl A, et al. Hospital readmissions and emergency department visits in moderate preterm, late preterm, and early term infants. Clin Perinatol 2013;40:753-75.

10 Kuint J, Lerner-Geva L, Chodick G, et al. Rehospitalization through childhood and adolescence: association with neonatal morbidities in infants of very low birth weight. J Pediatr 2017;188:135-41.

11 Melville JM, Moss TJM. The immune consequences of preterm birth. Front Neurosci 2013;7:79.

12 Laugier O, Garcia P, Boucékine M, et al. Influence of socioeconomic context on the rehospitalization rates of infants born preterm. $J$ Pediatr 2017;190:174-9.

13 Petrou S, Eddama O, Mangham L. A structured review of the recent literature on the economic consequences of preterm birth. Arch Dis Child Fetal Neonatal Ed 2011;96:F225-32.

14 Wang ML, Dorer DJ, Fleming MP, et al. Clinical outcomes of nearterm infants. Pediatrics 2004;114:372-6.

15 Schmitt SK, Sneed L, Phibbs CS. Costs of newborn care in California: a population-based study. Pediatrics 2006;117:154-60.

16 Shapiro-Mendoza CK, Tomashek KM, Kotelchuck M, et al. Risk factors for neonatal morbidity and mortality among "healthy," late preterm newborns. Semin Perinatol 2006;30:54-60.

17 Gladstone M, Oliver C, Van den Broek N. Survival, morbidity, growth and developmental delay for babies born preterm in low and middle income countries - a systematic review of outcomes measured. PLoS One 2015;10:e0120566.

18 Ballard JL, Khoury JC, Wedig K, et al. New ballard score, expanded to include extremely premature infants. J Pediatr 1991;119:417-23.

19 Engle WA. American Academy of pediatrics committee on fetus and newborn HJ. Age terminology during the perinatal period. Pediatrics 2004;114:1362-4.

20 Do CHT, Kruse AY, Wills B, et al. Neurodevelopment at 2 years corrected age among Vietnamese preterm infants. Arch Dis Child 2020;105:134-40.

21 Mourani PM, Kinsella JP, Clermont G, et al. Intensive care unit readmission during childhood after preterm birth with respiratory failure. J Pediatr 2014;164:749-55.

22 Vohr B, McGowan E, Keszler L, et al. Impact of a transition home program on rehospitalization rates of preterm infants. J Pediatr 2017:181:86-92.

23 Elder DE, Hagan R, Evans SF, et al. Hospital admissions in the first year of life in very preterm infants. $J$ Paediatr Child Health 1999;35:145-50.

24 Lamarche-Vadel A, Blondel B, Truffer P, et al. Re-hospitalization in infants younger than 29 weeks' gestation in the EPIPAGE cohort. Acta Paediatr 2004:93:1340-5.

25 Luu TM, Lefebvre F, Riley P, et al. Continuing utilisation of specialised health services in extremely preterm infants. Arch Dis Child Fetal Neonatal Ed 2010;95:F320-5. 
26 Ambalavanan N, Carlo WA, McDonald SA, et al. Identification of extremely premature infants at high risk of rehospitalization. Pediatrics 2011;128:e1216-25.

27 Ralser E, Mueller W, Haberland C, et al. Rehospitalization in the first 2 years of life in children born preterm. Acta Paediatr 2012;101:e1-5.

28 Underwood MA, Danielsen B, Gilbert WM. Cost, causes and rates of rehospitalization of preterm infants. J Perinatol 2007;27:614-9.

29 Young PC, Korgenski K, Buchi KF. Early readmission of newborns in a large health care system. Pediatrics 2013;131:e1538-44.

30 Aykanat Girgin B, Cimete G. Rehospitalization of preterm infants according to the discharge risk level. J Spec Pediatr Nurs 2017;22:e12165.

31 McLaurin KK, Hall CB, Jackson EA, et al. Persistence of morbidity and cost differences between late-preterm and term infants during the first year of life. Pediatrics 2009;123:653-9.

32 Escobar GJ, Greene JD, Hulac P, et al. Rehospitalisation after birth hospitalisation: patterns among infants of all gestations. Arch Dis Child 2005;90:125-31.
33 Hong T, Bolisetty S, Bajuk B, et al. A population study of respiratory rehospitalisation in very preterm infants in the first 3 years of life. $J$ Paediatr Child Health 2016;52:715-21.

34 Walker CLF, Rudan I, Liu L, et al. Global burden of childhood pneumonia and diarrhoea. Lancet 2013;381:1405-16.

35 Hanieh S, Ha TT, Simpson JA, et al. Exclusive breast feeding in early infancy reduces the risk of inpatient admission for diarrhea and suspected pneumonia in rural Vietnam: a prospective cohort study. BMC Public Health 2015; 15:1166.

36 Ho NT, Thompson C, Nhan LNT, et al. Retrospective analysis assessing the spatial and temporal distribution of paediatric acute respiratory tract infections in Ho Chi Minh City, Vietnam. BMJ Open 2018;8:e016349.

37 Nguyen TKP, Nguyen DV, Truong TNH, et al. Disease spectrum and management of children admitted with acute respiratory infection in Viet Nam. Trop Med Int Health 2017;22:688-95.

38 Bhutta ZA, Das JK, Walker N, et al. Interventions to address deaths from childhood pneumonia and diarrhoea equitably: what works and at what cost? Lancet 2013;381:1417-29. 